\title{
Indicadores de bienestar animal para detectar problemas en el cajón de insensibilización de bovinos
}

\author{
Animal welfare indicators to detect problems in the cattle stunning box \\ D Muñoz, A Strappini, C Gallo*
}

Instituto de Ciencia Animal, Facultad de Ciencias Veterinarias, Universidad Austral de Chile, Valdivia, Chile.

\begin{abstract}
SUMMARY
The aim of this study was to identify animal welfare problems during the stunning process in a beef slaughtering plant. Animal welfare indicators such as behaviour and handling by the personnel, were evaluated on 1,025 cattle (cows, heifers and steers) during five days of commercial slaughter; cattle were stunned with a non-penetrating captive bolt pistol. A descriptive analysis was used to show the frequencies of the indicators and a Chi-square test to determine the association between some of them. The most frequent behaviours in cattle were struggling (38.3\%), vocalization (17.2\%) and falling (9.5\%). The guillotine door hit $75.1 \%$ of cattle on entering the stunning box. On $49.2 \%$ of cattle an electric prod was used. Prodding was significantly associated with vocalization $(\mathrm{P}<0.001)$, falling $(\mathrm{P}=0.02)$ and escape attempts $(\mathrm{P}<0.001)$. Most cattle were effectively restrained at the first attempt $(92.3 \%)$; however in $6.1 \%$ of the cases the effective restraint was incorrect, which was significantly associated with vocalization $(\mathrm{P}<0.001)$ and falling $(\mathrm{P}=0.01)$. These results, together with the fact that in only $86.7 \%$ of cattle loss of posture was attained after the first shot indicate that there are animal welfare problems during stunning at this plant. It is concluded that some changes in the structure of the stunning box, as well as training of personnel and closer supervision of the process could help in solving the problems found.
\end{abstract}

Palabras clave: proceso de insensibilización, manejo, comportamiento bovino.

Key words: stunning process, handling, cattle behaviour.

\section{INTRODUCCIÓN}

A pesar del creciente interés mundial respecto al bienestar de los animales de producción (Stuardo 2005), en Chile se ha dado escasa atención a la relación entre la infraestructura de manejo de los bovinos y su bienestar, existiendo algunos antecedentes para el caso de vacas lecheras (Córdoba y col 2012). En el ganado de carne, las condiciones de manejo antes del sacrificio sugieren un alto riesgo para el bienestar animal, en especial el proceso de insensibilización, al ser uno de los últimos procedimientos ante-mortem a que se someten los animales (Gallo 2009).

El objetivo de la insensibilización o noqueo es lograr una pérdida profunda de la conciencia del animal, evitando cualquier sufrimiento del mismo durante el desangrado (HSA 2006). En Chile, la insensibilización es de carácter obligatorio (MINAGRI 2009), y la mayoría de las plantas de sacrificio de ganado bovino emplean la pistola de proyectil retenido, con o sin penetración de cráneo, como método de noqueo (Gallo y col 2003).

Para evaluar el bienestar animal existen métodos directos y métodos indirectos (Broom 1991); los primeros evalúan variables conductuales y fisiológicas de los animales así como la condición física de éstos, mientras que los indicadores

Aceptado: 24.05.2012.

* Casilla 567, Valdivia, Chile; cgallo@uach.cl indirectos evalúan el ambiente o infraestructura en que se encuentran los animales, así como el manejo que reciben por parte de las personas en los lugares de producción, comercialización (ferias), transporte y plantas de sacrificio.

El registro de vocalizaciones es un ejemplo de método directo, debido a que esta conducta puede ser indicadora de dolor, miedo o estrés en los animales y puede presentarse en respuesta a estímulos como la aplicación de la picana eléctrica (Grandin 1998'a). A su vez, el excesivo uso de picana habitualmente se observa en plantas de sacrificio con deficiencias en el diseño y/o mantención de sus equipos e instalaciones (Grandin 2000, 2007). Altamirano (2004) en un estudio en una planta de sacrificio chilena identificó que el uso de la picana eléctrica, además, resultaba ser un estímulo generador de reacciones violentas en los bovinos, constituyendo uno de los principales factores de resbalones y caídas de los animales en la manga de acceso y en el cajón de noqueo.

La existencia de un sistema de sujeción para los bovinos en el cajón de noqueo ayuda a mejorar la efectividad del proceso (Gallo y col 2003) y es actualmente obligatoria según la reglamentación chilena (MINAGRI 2009). Sin embargo, este manejo puede resultar estresante para el ganado si no se efectúa correctamente (Ewbank y col 1992, OIE 2011). Estudios de Grandin (2000) y Bourguet y col (2011) han demostrado que la sujeción incrementa el número de animales que vocaliza, y que esta conducta podría reflejar una presión excesiva sobre el animal en determinados casos. 
Por lo anterior, se recomienda insensibilizar al animal sin dilación una vez aplicado el dispositivo de sujeción (HSA 2006, OIE 2011), de manera de mantener sujeto al animal sólo durante unos pocos segundos.

El animal debe quedar insensible al primer disparo (HSA 2006, OIE 2011). Algunas razones de la inefectividad del noqueo son la falta de capacitación del personal (OIE 2011) y factores estructurales y de equipamiento. Al respecto Gallo y col (2003) encontraron mejorías en la eficacia del noqueo tras la implementación conjunta de un dispositivo de sujeción y una pistola de noqueo con compresor de aire exclusivo, además de la capacitación del personal a cargo del proceso.

El objetivo de este estudio fue identificar problemas de bienestar animal durante el proceso de insensibilización en una planta de sacrificio de bovinos utilizando indicadores conductuales y de manejo en el cajón de noqueo, y determinar a su vez si existía relación entre tales indicadores.

\section{MATERIAL Y MÉTODOS}

El estudio se llevó a cabo en una planta de sacrificio del sur de Chile que procesa alrededor de 200 bovinos diarios (40 bovinos sacrificados/hora). La metodología comprendió un período preexperimental de observación de dos semanas donde se identificaron las características del cajón y el equipo de noqueo, y se definieron las variables conductuales a registrar en los bovinos y las variables relacionadas con los manejos por parte del noqueador que pudieran servir como indicadores de problemas de bienestar animal. Para la toma de datos se empleó básicamente la metodología de Grandin (1998b), quien señala que debe muestrearse un mínimo de cien animales en plantas de sacrificio grandes. Se observó un total de 1.025 bovinos durante los cinco días de sacrificio habitual de una semana, los que correspondieron a 212 vacas (jóvenes, adultas y viejas), 195 vaquillas y 618 novillos (novillitos y novillos), según la clasificación oficial del ganado bovino (INN 1994) efectuada por personal de la planta.

\section{CARACTERÍSTICAS GENERALES DEL CAJÓN DE NOQUEO Y SISTEMA DE NOQUEO}

El cajón de noqueo era de cemento, tenía una altura de $1,95 \mathrm{~m}$, un ancho de $91,5 \mathrm{~cm}$ y un largo de 2,80 $\mathrm{m}$, con una puerta de ingreso tipo guillotina (figura 1) y una puerta lateral de salida (sube y baja), ambas de acero inoxidable. Existía un espacio de $40 \mathrm{~cm}$ de alto en la parte inferior de la puerta lateral por donde entraba luz (figura 2). El piso era de cemento y presentaba una inclinación de $13^{\circ}$ hacia la puerta lateral para facilitar la extracción del animal insensibilizado. El sistema de sujeción, ubicado en la parte anterior del cajón, era de acero inoxidable y consistía en dos barras verticales que sujetaban el cuello del animal más una plataforma para elevar el mentón (figura 1). La puerta lateral del cajón y el sistema de sujeción funcionaban con

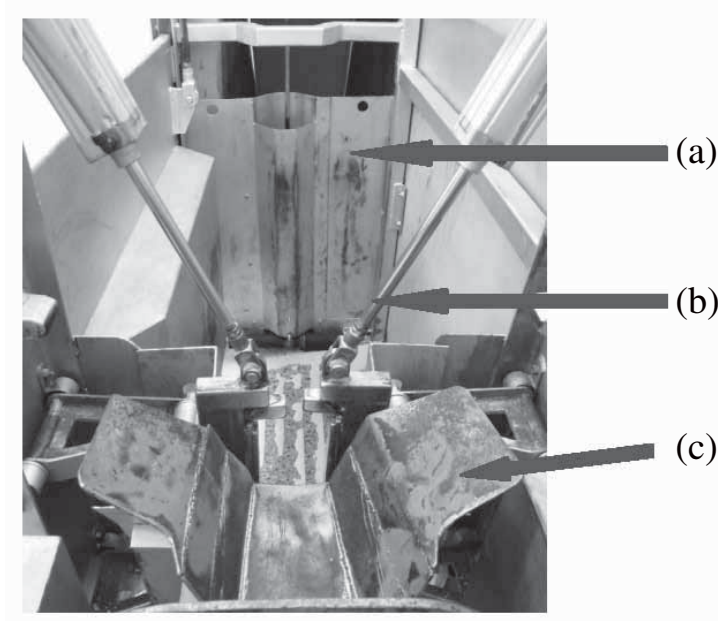

Figura 1. Vista del interior del cajón de noqueo, mostrando la puerta de ingreso tipo guillotina (a), el dispositivo de sujeción cervical (b) y el eleva mentón (c).

Inside view of the stunning box showing the guillotine door (a), neck restrainer (b) and head holder (c).

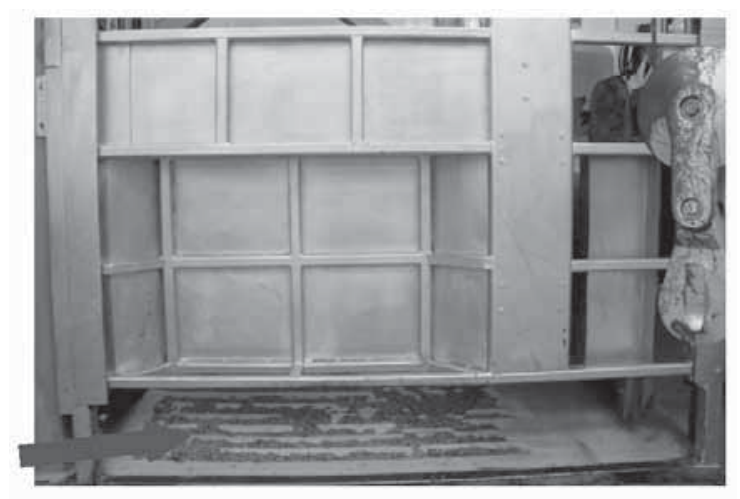

Figura 2. Vista lateral del cajón de noqueo, mostrando el espacio dejado bajo la puerta lateral.

Lateral view of the stunning box showing the space left under the lateral door.

aire comprimido, suministrado por un compresor de aire compartido. El equipo para insensibilizar correspondía a una pistola neumática de proyectil retenido sin penetración, marca JARVIS modelo USSS-2 que funcionaba con aire comprimido entregado por un compresor exclusivo, regulable a tres niveles de presión: baja (165 psi), media (185 psi) y alta (215 psi).

Los indicadores de bienestar animal se registraron para cada animal ingresado al cajón de noqueo, en el tiempo comprendido entre el cierre de la puerta de entrada del cajón tipo guillotina resultando con el animal dentro del cajón, hasta el primer disparo en el cráneo del animal. Los indicadores fueron registrados como presentes cuando se observaron al menos una vez en cada animal y fueron definidos de la siguiente forma: 
INDICADORES DIRECTOS O DE COMPORTAMIENTO

Vocalización: el animal emite un mugido.

Caída: el animal se desequilibra y una parte del cuerpo diferente a las pezuñas toca el piso.

Forcejeo: mientras la cabeza y cuello del animal permanecen sujetos por el dispositivo de sujeción, el cuerpo de éste se sacude con movimientos bruscos de un lado hacia otro.

Intentos de fuga: el animal realiza movimientos para buscar una salida en el cajón de noqueo. Las siguientes tres conductas fueron clasificadas como intentos de fuga: a) trepar: el animal se para en sus dos miembros posteriores y apoya uno o ambos miembros anteriores sobre una pared del cajón de noqueo o sobre el dispositivo de sujeción; b) giro: el animal en posición de pie dentro del cajón de noqueo da media vuelta o una vuelta completa sobre sí mismo; c) intento de escape bajo la puerta lateral: el animal baja la cabeza y la introduce parcial o completamente y acompañada o no de uno de sus miembros anteriores, en el espacio existente bajo la puerta lateral del cajón de noqueo.

\section{INDICADORES INDIRECTOS O DE MANEJO}

Golpe con la puerta tipo guillotina: el animal al ingresar al cajón de noqueo es golpeado por la puerta cuando ésta baja al ser accionada por un operario.

Aplicación de picana: el animal recibe una descarga eléctrica cuando el electrodo de la picana eléctrica, accionada por el operario, entra en contacto con cualquier parte del cuerpo del animal.

Sujeción: el operario aplica el dispositivo de sujeción en cualquier parte del cuerpo del animal. Se registró el número de animales sujetos al primer intento sin repetir la sujeción. Además se caracterizó la sujeción efectiva como sujeción efectiva correcta cuando el dispositivo de sujeción fue aplicado sólo en el cuello del animal y sujeción efectiva incorrecta cuando el dispositivo fue aplicado en otra parte del cuerpo distinta al cuello, o en el cuello más alguna extremidad.

Eficiencia en producir la pérdida de postura al primer disparo: el animal cae tras el primer disparo.

Tiempo total de permanencia en el cajón: tiempo que transcurre desde que la puerta tipo guillotina cae detrás o sobre el animal hasta el primer disparo.

Tiempo de sujeción: tiempo que transcurre desde que se logra la sujeción efectiva del animal hasta que se realiza el primer disparo.

\section{ANÁLISIS ESTADÍSTICO}

Se obtuvieron las frecuencias (n y \%) de todos los indicadores para el total de bovinos y por categoría. Se determinó si existía asociación entre distintos indicadores usando una prueba de Chi cuadrado con un nivel de significancia de 0,05 , para lo cual se utilizó el programa estadístico Statistix-8. El porcentaje total de animales que vocalizó, cayó, se le aplicó picana y perdió la postura de pie tras al primer disparo, se comparó con los estándares utilizados para evaluar el bienestar animal en plantas faenadoras según Grandin (1998b). Para el caso del tiempo de sujeción y tiempo total de permanencia en el cajón se determinó el promedio para cada indicador. Para poder asociar el tiempo de sujeción (variable continua) a los indicadores de comportamiento (categóricos), dicho tiempo se categorizó arbitrariamente en "hasta 5 segundos" o "mayor a 5 segundos", considerando que este es un tiempo suficiente para que se efectúe el disparo una vez sujeto el animal.

\section{RESULTADOS Y DISCUSIÓN}

De los indicadores de comportamiento, el forcejeo fue la conducta más frecuentemente observada, registrándose en un 38,3\% de los animales (cuadro 1) y su presentación se asoció significativamente $(\mathrm{P}<0,001)$ con un tiempo

Cuadro 1. Número y porcentaje de bovinos (en total y por categoría) en que se registraron las conductas indicadas en el cajón de noqueo. Number and percentage of cattle (in total and by category) in which the different behaviours were recorded in the stunning box.

\begin{tabular}{|c|c|c|c|c|c|c|c|c|}
\hline \multirow{2}{*}{ Conductas } & \multicolumn{2}{|c|}{$\begin{array}{c}\text { Total } \\
\mathrm{n}=1.025\end{array}$} & \multicolumn{2}{|c|}{$\begin{array}{c}\text { Novillos } \\
\mathrm{n}=618\end{array}$} & \multicolumn{2}{|c|}{$\begin{array}{c}\text { Vaquillas } \\
\mathrm{n}=195\end{array}$} & \multicolumn{2}{|c|}{$\begin{array}{c}\text { Vacas } \\
\mathrm{n}=212\end{array}$} \\
\hline & $\mathrm{N}^{\mathrm{o}}$ & $\%$ & $\mathrm{~N}^{\mathrm{o}}$ & $\%$ & $\mathrm{~N}^{\mathrm{o}}$ & $\%$ & $\mathrm{~N}^{\mathrm{o}}$ & $\%$ \\
\hline Vocalización & 176 & 17,2 & 80 & 12,9 & 66 & 33,8 & 30 & 14,2 \\
\hline Caída & 97 & 9,5 & 50 & 8,1 & 38 & 19,5 & 9 & 4,2 \\
\hline Forcejeo & 393 & 38,3 & 224 & 36,2 & 122 & 62,6 & 47 & 22,2 \\
\hline \multicolumn{9}{|l|}{ Intentos de fuga } \\
\hline Trepar & 57 & 5,6 & 25 & 4 & 25 & 12,8 & 7 & 3,3 \\
\hline Escape bajo la puerta & 26 & 2,5 & 16 & 2,6 & 9 & 4,6 & 1 & 0,5 \\
\hline Giro & 5 & 0,5 & 1 & 0,2 & 4 & 2,1 & 0 & 0 \\
\hline
\end{tabular}


de sujeción mayor a 5 segundos. Esta relación se explica porque mientras más demoraba el noqueador, más tendía a forcejear el animal. Los novillos y especialmente las vaquillas tuvieron la mayor presentación de esta conducta (cuadro 1), lo que podría explicarse por el menor tamaño de estos animales en comparación a las vacas; al ser más pequeños tenían menos puntos de apoyo en las paredes del cajón de noqueo quedando más sueltos.

El 17,2\% de los animales vocalizó en el cajón de noqueo (cuadro 1), siendo este porcentaje superior al 10\% registrado por Altamirano (2004) en esta misma planta durante el arreo por la manga desde el pasillo principal de los corrales al cajón de noqueo. Según los estándares para evaluar el bienestar animal propuestos por Grandin $\left(1998^{b}\right)$, el alto porcentaje de animales que vocalizó refleja un "problema grave", distando bastante del 3\% máximo aceptable para esta conducta en los mataderos comerciales evaluados por este autor.

La OIE (2011) señala que debe evitarse la presión excesiva en los sistemas de sujeción para la insensibilización. Debido a que el dispositivo de sujeción se cerraba de forma uniforme y a igual presión para todos los animales, su aplicación en partes del cuerpo de mayor grosor que el cuello, lo que ocurrió en el 6,1\% de los animales efectivamente sujetos (cuadro 2), significaba una mayor presión sobre el animal y una sujeción incorrecta. La asociación significativa $(\mathrm{P}<0,001)$ encontrada entre la 'sujeción efectiva incorrecta' y la 'vocalización', sumado al conocimiento de que esta conducta puede ser una respuesta frente al dolor (Grandin 1998 a , 2000), indica que la presión excesiva puede ser fuente de dolor en los animales evaluados y parte de las vocalizaciones podrían deberse a este hecho. Una capacitación del noqueador para una sujeción adecuada así como la posibilidad de regular la presión del dispositivo de sujeción deberían contribuir a reducir los niveles de vocalización e incomodidad.

La aplicación de la picana eléctrica para acomodar a los animales en el sistema de sujeción dentro del cajón de noqueo se asoció significativamente $(\mathrm{P}<0,001)$ a las vocalizaciones, lo que estaría indicando que este manejo también puede ser una fuente de dolor. Consecuentemente, las vaquillas, que fueron la categoría bovina más picaneada (cuadro 2), mostraron un alto porcentaje de vocalizaciones $(33,8 \%$, cuadro 1$)$.

El 9,5\% de los bovinos cayó en el cajón de noqueo (cuadro 1), lo que refleja un "problema grave" de bienestar animal, ya que tal resultado dista bastante del $1 \%$ máximo aceptado por Grandin $\left(1998^{\mathrm{b}}\right)$. El hecho de que un elevado porcentaje de animales cayera se debió en parte a que el piso no era suficientemente antideslizante, como lo exige el Reglamento Sobre Estructura y Funcionamiento de Mataderos (MINAGRI 2009), y a las reacciones bruscas de movimiento de los animales frente al uso excesivo de la picana eléctrica (cuadro 2). Por otro lado, se encontró asociación significativa $(\mathrm{P}=0,01)$ entre la sujeción efectiva incorrecta y la caída de los animales. Esto ocurría cuando la sujeción incluía alguna extremidad y por tanto los animales tendían a perder el equilibrio y caer. Las vaquillas fueron las que más cayeron (cuadro 1), lo que podría explicarse en parte porque justamente en ellas ocurrió el mayor porcentaje de animales sujetos incorrectamente (cuadro 2).

Los intentos de fuga, aunque menos frecuentes que las vocalizaciones y las caídas (cuadro 1), reflejaron problemas de infraestructura en el cajón de noqueo. En primer lugar el cajón era muy ancho, lo que permitía que animales pequeños como novillos y vaquillas giraran dentro de éste. Grandin (2007) señala que un cajón de noqueo de $76 \mathrm{~cm}$ de ancho es suficiente para mantener cualquier tipo de bovino, excepto toros y bueyes muy grandes. Dada la alta variabilidad en el tamaño de los bovinos sacrificados en Chile, una posible solución sería que la pared lateral del cajón de noqueo fuera ajustable y permitiera presionar lateralmente el cuerpo de los animales, procurando evitar presión excesiva y usar un material blando, que se ajuste al contorno del cuerpo del animal, tipo bolsas de aire ("airbag"). Por otro lado, se identificaron dos rutas de escape que constituyeron distractores para los animales (Grandin 1996): una era el espacio que había bajo la puerta lateral del cajón de noqueo, que al dejar entrar luz incentivó especialmente a vaquillas y novillos (de menor tamaño) a intentar escapar por allí, y otra la pared frontal del cajón, la cual, al no ser lo suficientemente alta

Cuadro 2. Número y porcentaje de bovinos (en total y por categoría) sometidos por parte del personal a los manejos indicados en el cajón de noqueo.

Number and percentage of cattle (in total and by class) submitted to the handling procedures indicated in the stunning box by the staff.

\begin{tabular}{|c|c|c|c|c|c|c|c|c|}
\hline \multirow[t]{2}{*}{ Manejos } & \multicolumn{2}{|c|}{$\begin{array}{c}\text { Total } \\
\mathrm{n}=1.025\end{array}$} & \multicolumn{2}{|c|}{$\begin{array}{c}\text { Novillos } \\
\mathrm{n}=618\end{array}$} & \multicolumn{2}{|c|}{$\begin{array}{c}\text { Vaquillas } \\
\mathrm{n}=195\end{array}$} & \multicolumn{2}{|c|}{$\begin{array}{c}\text { Vacas } \\
\mathrm{n}=212\end{array}$} \\
\hline & $\mathrm{N}^{\mathrm{o}}$ & $\%$ & $\mathrm{~N}^{\mathrm{o}}$ & $\%$ & $\mathrm{~N}^{\mathrm{o}}$ & $\%$ & $\mathrm{~N}^{\mathrm{o}}$ & $\%$ \\
\hline Golpe con la puerta de guillotina & 770 & 75,1 & 462 & 74,8 & 125 & 64,1 & 183 & 86,3 \\
\hline Aplicación de picana eléctrica & 504 & 49,2 & 263 & 42,6 & 180 & 92,3 & 61 & 28,8 \\
\hline Sujeción al $1^{\mathrm{er}}$ intento & 946 & 92,3 & 573 & 92,7 & 172 & 88,2 & 201 & 94,8 \\
\hline Sujeción efectiva incorrecta & 63 & 6,1 & 37 & 6 & 16 & 8,2 & 10 & 4,7 \\
\hline Pérdida postura al $1^{\mathrm{er}}$ disparo & 889 & 86,7 & 527 & 85,3 & 176 & 90,3 & 186 & 87,7 \\
\hline
\end{tabular}


permitía que algunos animales intentaran saltar por ahí, empleando el dispositivo de sujeción como peldaño para escapar. Eliminar estas rutas de escape deberían reducir los intentos de fuga.

El 75,1\% de los animales fue golpeado por la puerta tipo guillotina (cuadro 2) al ingreso al cajón de noqueo. Esto se debió básicamente a que el noqueador usaba esta puerta como un mecanismo para acelerar el ingreso de los animales al cajón y a la vez evitar el ingreso de un segundo animal, dejándola caer sobre el animal, golpeándolo. Por otra parte, ocurría que en los animales de gran tamaño al ingresar al cajón parte del cuerpo quedaba fuera de éste, siendo inevitablemente golpeados por la puerta. Estos animales correspondieron en su mayoría a vacas de descarte de lechería, de gran tamaño, y esto explica por qué se encontró un alto porcentaje de vacas golpeadas por esta puerta (cuadro 2).

La OIE (2011) señala que la picana eléctrica deberá usarse sólo en casos extremos y no de manera rutinaria. Sin embargo el 49,2\% de los animales resultó picaneado (cuadro 2), lo que se aleja bastante del $20 \%$ máximo aceptado por Grandin $\left(1998^{\mathrm{b}}\right)$. También Cáraves y col (2006) registraron un promedio de $41,8 \%$ de bovinos en que se utilizó picana eléctrica para hacer ingresar los animales al cajón de noqueo en las seis principales plantas de sacrificio de Chile, lo que refleja que es un problema generalizado y no sólo de esta planta. Las vaquillas fueron la clase bovina en que se observó el mayor porcentaje de uso de picana eléctrica (cuadro 2) por parte del operario. Como consecuencia de esto algunos animales vocalizaron, cayeron o intentaron fugarse. Se encontró una asociación significativa entre la aplicación de picana eléctrica y la vocalización $(\mathrm{P}<0,001)$, las caídas $(\mathrm{P}=0,02)$ y los intentos de fuga $(\mathrm{P}<0,001)$.

La sujeción en sí misma suele ser estresante para los animales (Ewbank y col 1992, HSA 2006) y lo es mucho más cuando éstos se exponen a excesivo roce o presión con el dispositivo de sujeción (Grandin 1998a), como ocurre en sujeciones infructuosas y reiteradas. Si bien no hay un indicador estandarizado en cuanto al porcentaje de animales que es aceptable sujetar al primer intento, el $92,3 \%$ de los animales sujetos al primer intento en este estudio (cuadro 2) resultó superior al 38,2\% registrado por Ewbank y col (1992); no obstante, en ambos casos el porcentaje logrado implicó que un elevado número de animales debieron ser persuadidos a colocar el cuello en el sistema de sujeción usando picana eléctrica, es decir, no ingresaron voluntariamente.

Los animales una vez sujetos deben ser aturdidos sin dilación (OIE 2011), aunque no se señala un tiempo considerado como máximo. En este estudio se obtuvo un tiempo promedio de sujeción de 14 segundos, lo que se debió a que en general se ingresaba el animal al cajón de noqueo y sujetaba cuando el noqueador aún no estaba listo para su labor. Gallo y col (2003) indican que no se debería introducir un bovino en el cajón y/o fijar su cabeza antes de que el noqueador esté listo para un disparo certero y que haya además un operario listo para realizar el desangrado. A pesar de lo anterior, el tiempo total promedio de permanencia de los animales en el cajón de noqueo fue de 21,1 segundos, menor que los 34,2 segundos encontrados por Ewbank y col (1992).

Daly (1985) señala que cualquier método de insensibilización debe ser instantáneo; sin embargo, en este estudio sólo el $86,7 \%$ de animales perdió postura (cayó) al primer disparo, lo que está lejos del 95\% considerado por Grandin $\left(1998^{\mathrm{b}}\right)$ como mínimo aceptable. Por su parte, Gallo y col (2003) registraron anteriormente un 97,8\% de animales noqueados al primer disparo en esta misma planta, pero empleando una pistola de proyectil retenido con penetración e inmediatamente posterior a una capacitación del personal en el uso de este equipo. La mayor probabilidad de fallas de la pistola de proyectil retenido no penetrante (OIE 2011) y que el noqueador no haya sido capacitado para usar este tipo de pistola específicamente, podrían explicar las diferencias encontradas entre ambos estudios. La pistola de proyectil retenido no penetrante requiere una ubicación $1 \mathrm{~cm}$ por encima de la recomendada para la pistola de proyectil retenido penetrante, además de distintas presiones de aire según el tamaño de los animales y un desangrado dentro de 20 segundos (OIE 2011).

Según la HSA (2006) la insensibilización del animal con pistola neumática se logra usando una presión del aire adecuada. En esta planta el noqueador escogía el nivel de presión a usar tras estimar la edad del animal por medio de una inspección de éste y tras consultar el programa diario de faena, el cual otorgaba información de la edad y sexo de cada lote de animales a sacrificar. Sin embargo, esta información en muchos casos no fue concordante con la categorización efectuada por el certificador de carne. Esto indicaría que la ineficacia de la insensibilización en algunos casos podría deberse al uso de una presión de aire insuficiente en la pistola por un error en la estimación de la edad de los animales.

De este estudio se puede concluir que hay problemas que pueden afectar el bienestar de los animales durante el proceso de insensibilización y que éstos se pueden detectar usando como indicadores el comportamiento animal y los manejos realizados por los operarios. La relación encontrada entre algunos indicadores directos e indirectos permite concluir que mejorando la infraestructura del cajón de noqueo, capacitando al noqueador y efectuando una constante supervisión del proceso de insensibilización es posible reducir los problemas. Por otro lado, incluir un sistema de sujeción de cuerpo completo, ajustable al tamaño del bovino, podría disminuir el número de animales que giran y forcejean dentro de éste. Instalar un piso antideslizante y reducir el espacio dejado bajo la puerta lateral del cajón disminuiría el porcentaje de animales que cae y que intentan fugarse, facilitando el manejo de los animales, la labor del noqueador y la eficacia del proceso. Además, desde el punto del bienestar animal se 
recomienda usar para la insensibilización un sistema de noqueo irreversible, como la pistola de proyectil retenido con penetración de cráneo, ya que como lo menciona la OIE (2011) tiene menor probabilidad de falla que los métodos de noqueo reversibles.

\section{RESUMEN}

El objetivo del estudio fue identificar problemas de bienestar animal durante el proceso de insensibilización en una planta de sacrificio de bovinos. Se evaluaron indicadores de bienestar animal, como conductas de los bovinos y manejos por parte del personal en el cajón de noqueo, en 1.025 bovinos de las tres principales categorías sacrificadas (vacas, vaquillas y novillos) durante cinco días de faena comercial; los animales fueron insensibilizados con pistola de proyectil retenido sin penetración. Se describieron frecuencias de los indicadores y se usó una prueba de Chi cuadrado para determinar asociación entre algunos de ellos. Las conductas más frecuentes fueron forcejeo $(38,3 \%)$, vocalización $(17,2 \%)$ y caída $(9,5 \%)$. Un $75,1 \%$ de los bovinos fue golpeado por la puerta tipo guillotina al entrar al cajón y el $49,2 \%$ fue picaneado. El picaneo se asoció significativamente a la vocalización $(\mathrm{P}<0,001)$, caída $(\mathrm{P}=0,02)$ e intentos de fuga $(\mathrm{P}<0,001)$. El 92,3\% de los animales fue sujeto al primer intento; sin embargo en un $6,1 \%$ la sujeción efectiva fue incorrecta, lo que se asoció significativamente con vocalizaciones $(\mathrm{P}<0,001)$ y caídas $(\mathrm{P}=0,01)$. Estos resultados, y el hecho de que sólo en un $86,7 \%$ de los bovinos se logró la pérdida de la postura de pie tras el primer disparo indican que existen problemas de bienestar animal durante el proceso de insensibilización en la planta. Se concluye que algunos cambios de infraestructura del cajón de noqueo, así como capacitación del noqueador y mayor vigilancia del proceso, permitirían superar los problemas encontrados.

\section{AGRADECIMIENTOS}

Los autores agradecen al personal de la planta faenadora donde se realizó este estudio y en especial a los doctores Héctor Mimica y Jorge Gasic por su gran disposición para la realización del mismo.

\section{REFERENCIAS}

Altamirano A. 2004. Evaluación del bienestar animal mediante la observación de tres indicadores en una planta faenadora de carne de bovino. Memoria de título, Escuela de Medicina Veterinaria, Universidad Austral de Chile, Valdivia, Chile.

Bourguet C, V Deiss, CC Tannugi C Terlouw. 2011. Behavioural and physiological reactions of cattle in a commercial abattoir: Relationships with organisational aspects of the abattoir and animal characteristics. Meat Sci 88, 158-168.

Broom DM. 1991. Animal welfare: concepts and measurement. $J$ Animal Sci 69, 4167-4175.

Cáraves M, C Gallo, A Strappini, L Aguayo, A Barrientos, R Allende, F Chacón, I Briones. 2006. Evaluación del bienestar animal de bovinos durante el manejo ante mortem en seis plantas faenadoras en Chile. Resúmenes de la XXXI Reunión Anual de SOCHIPA, Chillán, Chile, Pp 179-180.

Córdoba JD, MP Castillo, N Ormeño, G Acosta, N Tadich. 2012. Descripción de los cubículos utilizados en granjas lecheras en el sur de Chile y su relación con el confort de las vacas. Arch Med Vet $44,75-80$.

Daly C. 1985. Recent developments in captive bolt stunning. Humane slaughter of animals for food. Universities Federation for Animal Welfare (UFAW), Potters Bar, Herts, England.

Ewbank R, MJ Parker, CW Mason. 1992. Reactions of cattle to head restraint at stunning: A practical dilemma. Anim Welf 1, 55-63.

Gallo C, C Teuber, M Cartes, H Uribe, T Grandin. 2003. Mejoras en la insensibilización de bovinos con pistola neumática de proyectil retenido tras cambios de equipamiento y capacitación del personal. Arch Med Vet 35, 159-170.

Gallo C. 2009. Bienestar animal y buenas prácticas de manejo animal relacionadas con la calidad de la carne. En: Bianchi G, Feed O (eds). Introducción a la ciencia de la carne. Editorial Hemisferio Sur, Montevideo, Uruguay, Pp 455-494.

Grandin T. 1996. Bienestar animal en las plantas de faena. American Association of Bovine Practitioners, Proceedings, 22-26.

Grandin T. $1998^{\mathrm{a}}$. The feasibility of using vocalization scoring as an indicator of poor welfare during cattle slaughter. Appl Anim Behav Sci 56, 121-128.

Grandin T. $1998^{\mathrm{b}}$. Buenas prácticas de manejo para el arreo e insensibilización de animales. Informativo sobre carne y productos cárneos $\mathrm{N}^{\circ} 22,124-136$.

Grandin T. 2000. Effect of animal welfare audits of slaughter plants by a major fast food company on cattle handling and stunning practices. J Am Vet Med Assoc 216, 848-851.

Grandin T. 2007. Handling and welfare of livestock in slaughter plants. In: Grandin T (ed). Livestock handling and transport. $3^{\text {rd }}$ ed. CAB International, Wallingford, UK, Pp 329-353.

HSA, Humane Slaughter Association. 2006. Insensibilización del ganado con pistola neumática de perno cautivo. $4^{\mathrm{a}} \mathrm{ed}$. Wheathampstead, UK, Pp 1-13.

INN, Instituto Nacional de Normalización de Chile. 1994. Norma Chilena Oficial de Clasificación de ganado bovino, NCh 1423.

MINAGRI, Ministerio de Agricultura de Chile. 2009. Reglamento sobre estructura y funcionamiento de mataderos, establecimientos frigoríficos, cámaras frigoríficas y plantas de desposte, y fija equipamiento mínimo para tales establecimientos. Decreto $\mathrm{N}^{\circ} 94$ del 26 de noviembre de 2008, publicado en el Diario Oficial el 2 de junio de 2009.

OIE, Organización Mundial de Sanidad Animal. 2011. Código Sanitario para los animales terrestres, 2011. Directrices para el sacrificio de animales destinados al consumo humano. Editorial OIE, Paris, Francia.

Stuardo L. 2005. Chile, frente al desafío de la aplicación de normas de bienestar animal: la experiencia del Servicio Agrícola y Ganadero (SAG). Proceeding of the Seminar Animal Welfare in Chile and the EU: Shared Experiences and Future Objectives, Silvi Marina, Italy, Pp 41-46. 